\title{
Physiology of the distal convoluted tubule and collecting duct
}

\section{KK Purbhoo}

Department of Anaesthesia, Helen Joseph Hospital, University of the Witwatersrand, South Africa

Corresponding author, email: kashmirapurbhoo@gmail.com

\section{Summary}

Each human kidney contains approximately 1.3 million nephrons that are made up of a glomerulus and a renal tubule. The components of the renal tubule include the proximal convoluted tubule (PCT), the loop of Henle (LOH), the distal convoluted tubule (DCT) and the collecting duct (CD). Each component is made up of different cell subtypes that correlate with differences in function. ${ }^{1}$

The distal tubule follows the macula densa, and consists of two segments, the DCT and the connecting segment. The connecting segment extends to the CD which has a cortical and a medullary component. These segments have an important role in the absorption of many ions, and water reabsorption. ${ }^{2}$

The tubular fluid entering the distal tubule is hypotonic (100-200 mOsm). It contains about $20 \%$ of the water filtered by the glomerulus and about $10 \%$ of the sodium filtered. The early distal tubule reabsorbs a further $5 \%$ of the sodium, and the late distal tubule and CD fine tune reabsorption of the last little bit (around 5\%), determining exactly how much sodium will be excreted. ${ }^{3}$ The absolute level of transport of ions and water in these segments is much less than in most upstream segments, but the variability of the rate of transport is much higher. ${ }^{4}$ Sodium reabsorption in the late distal tubule and CD is regulated by hormones. ${ }^{1}$

In the context of renal tubule physiology, the term basolateral or serosal membrane refers to the cell membrane oriented away from the lumen of the tubule, whereas the term luminal or apical membrane refers to the cell membrane oriented towards the lumen.

Keywords: distal convoluted tubule, collecting duct, physiology

\section{Distal convoluted tubule (DCT)}

The DCT is approximately $5 \mathrm{~mm}$ long and can be further divided into two functionally distinct sub-segments, the early (DCT1) and the late (DCT2) DCTs. ${ }^{2}$

Although the DCT is the shortest segment of the nephron, it plays a critical role in a variety of homeostatic processes, including sodium chloride reabsorption, potassium secretion, and calcium and magnesium handling. ${ }^{2}$

Cells of the DCT have a unique morphology that matches their highly active physiology. They are among the most mitochondriarich in the kidney, indicating that the DCT engages in processes that require considerable ATP consumption and active transport of electrolytes driven by the basolateral $\mathrm{Na}^{+} / \mathrm{K}^{+}$-ATPase pump. ${ }^{2}$

\section{Early distal convoluted tubule}

The role of the early DCT is the absorption of ions, including sodium, chloride and calcium. Movement of all these ions is dependent on the function of $\mathrm{Na}^{+} / \mathrm{K}^{+}$ATPase transporter on the basolateral membrane of the cells. This excretes three sodium ions into the extracellular fluid and brings two potassium ions into the cell. This channel reduces intracellular sodium levels, creating a gradient which favours movement of sodium into the cell from the lumen of the DCT. As ATP is directly needed to set up the gradient, this process is referred to as primary active transport. ${ }^{2,5}$

Movement of sodium into the cell from the lumen of the DCT is chloride-dependent and mediated by thiazide-sensitive $\mathrm{NaCl}$ cotransporters expressed throughout the DCT (NCC symporter- $2^{\circ}$ active transport). Sodium and chloride transport through this cotransporter is interdependent, so inhibition of NCC by thiazide diuretics will also block chloride reabsorption in the DCT. ${ }^{2}$

The chloride ions then exit the cell through either a chloride ion uniporter or a potassium chloride cotransporter on the basolateral membrane into the extracellular fluid, preventing accumulation within the cell. The chloride ion uniporter is found throughout the DCT basolateral membrane and is called the $\mathrm{ClC}-\mathrm{Kb}$ channel. CIC-Kb channels require an accessory subunit called Barttin to be fully functional. The potassium chloride cotransporter 4 (KCC4) expressed in the basolateral membrane of the DCT mediates coupled electroneutral $\mathrm{K}-\mathrm{Cl}$ efflux ${ }^{2}$ (Figure 1).

Approximately $7-10 \%$ of filtered calcium is reabsorbed in the DCT. In contrast to other segments of the nephron, which passively reabsorb calcium through para-cellular routes, $100 \%$ of the calcium that is reabsorbed in the DCT occurs by active transcellular mechanisms under the influence of parathyroid hormone (PTH). Transient receptor potential vanilloid 5 (TRPV5) 


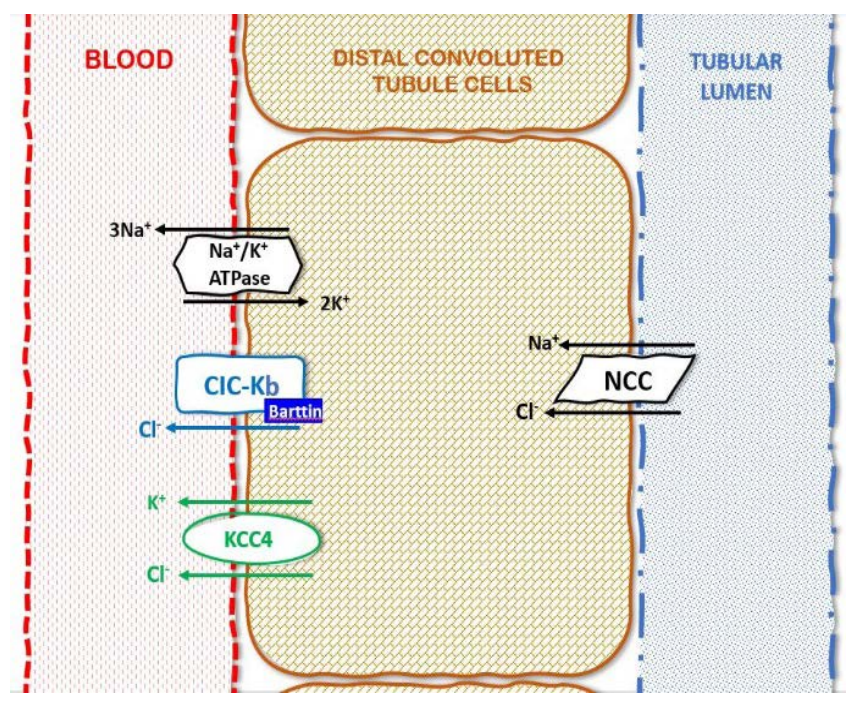

Figure 1: Early DCT cell

and 6 (TRPV6) in the apical membrane act as the main portal of entry for calcium, from the lumen into the DCT cell. ${ }^{2}$

PTH is released in response to low blood calcium levels from the parathyroid gland. PTH affects TRPV5 channel activity through multiple mechanisms:

1. It stimulates transcription of TRPV5.

2. It prevents TRPV5 degradation by inhibiting its removal from the apical surface of the DCT.

3. It stimulates direct phosphorylation of TRPV5 through a protein kinase A-dependent signalling pathway, which alters the gating characteristics of the channel, increasing the likelihood that it will be open. ${ }^{2}$

On entry, $\mathrm{Ca}^{2+}$ associates with the calcium binding protein calbindin, which buffers intracellular calcium levels and keeps free calcium concentrations low. After calcium is shuttled by calbindin to the basolateral surface, $\mathrm{Ca}^{2+}$ is extruded into the peritubular fluid by a sodium calcium exchanger ( $\mathrm{NCX}-2^{\circ}$ active

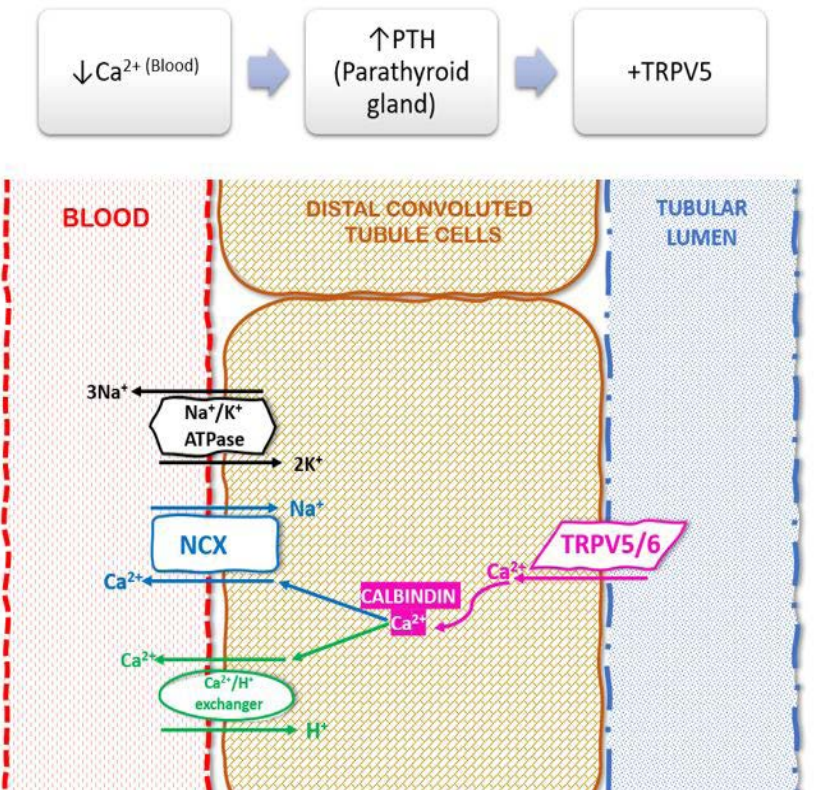

Figure 2: Calcium reabsorption transport), which utilises the sodium gradient established from the $\mathrm{Na}^{+} / \mathrm{K}^{+}$-ATPase channel, as well as by an ATP dependant calcium hydrogen exchanger. The reduction in intracellular calcium creates a gradient which draws calcium ions from the lumen of the tubule into the cell ${ }^{2,5}$ (Figure 2).

\section{Late distal convoluted tubule}

The early and late DCT can be distinguished by their differential responsiveness to the mineralocorticoid aldosterone. Aldosterone is a steroid hormone released from the adrenal gland in response to hyperkalaemia, angiotensin II (produced after the juxtaglomerular apparatus releases renin due to decreased blood pressure or low perfusion), hyponatraemia and corticotropin releasing hormone (which causes a small increase in aldosterone during stress). ${ }^{2}$

Aldosterone has a similar chemical structure to glucocorticoids, like cortisol, and both aldosterone and cortisol bind to the mineralocorticoid receptor with nearly equal affinity. Although mineralocorticoid receptors are expressed throughout the entire $D C T$, the late DCT principal cells are sensitive to the actions of aldosterone, because it expresses an enzyme called 11- $\beta$ hydroxysteroid dehydrogenase 2 (11- $\beta$ HSD2). ${ }^{2}$

$11-\beta H S D 2$ metabolises cortisol to the inactive metabolite cortisone, thereby preventing circulating glucocorticoids from binding to mineralocorticoid receptors expressed in the late DCT. Because the mineralocorticoid receptors in the late DCT can only be occupied by aldosterone, it is more sensitive than the early DCT to changes in circulating aldosterone levels. 11- $\beta$ HSD2 is also expressed in other downstream nephron segments, including the cortical collecting duct (CCD). For this reason, the late DCT, connecting tubule and CCD are collectively termed the aldosterone-sensitive distal nephron. ${ }^{2}$

Aldosterone mediates its effect in responsive cells by diffusing into the cell and binding cellular nuclear receptors which are then stimulated to increase mRNA synthesis. The mRNAs are then used to increase the expression of:

1. $\mathrm{Na}^{+} / \mathrm{K}^{+}$-ATPase pumps which are directed towards the basolateral membrane.

2. A renal outer medullary potassium channel (ROMK), which is directed towards the luminal surface.

3. An epithelial sodium channel ( $\mathrm{ENaC}$ ) directed towards the luminal surface. ${ }^{2,6}$

The $\mathrm{Na}^{+} / \mathrm{K}^{+}$-ATPase pumps, just like in the early DCT excretes three sodium ions into the extracellular fluid and brings two potassium ions into the cell. This channel reduces intracellular sodium levels, creating a gradient which favours movement of sodium into the cell from the lumen of the DCT via the ENaC channel. The $\mathrm{Na}^{+} / \mathrm{K}^{+}-$ATPase pumps also increases the intracellular potassium levels, creating a gradient which favours movement of potassium out of the cell into the lumen of the DCT via the ROMK channel (voltage dependant). As the activity of ENaC increases in the late DCT, more sodium is reabsorbed, 


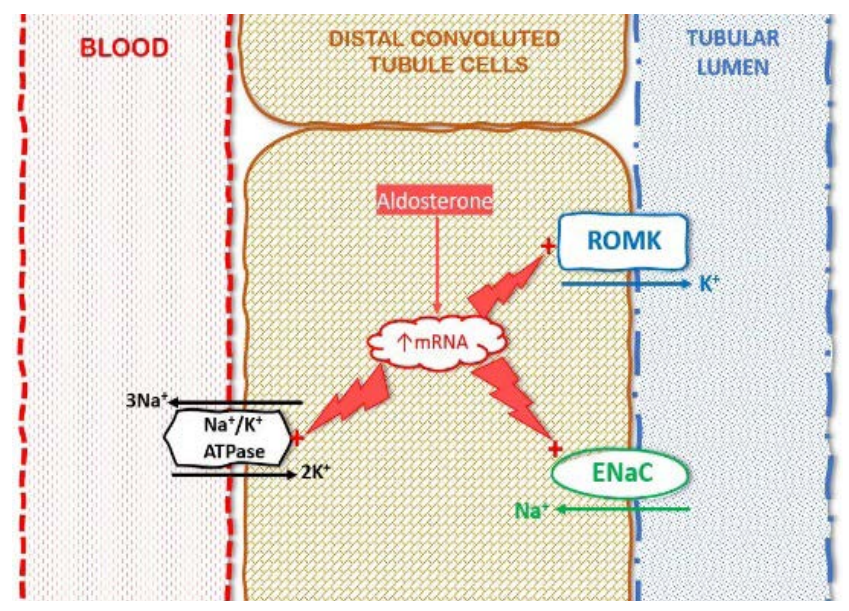

Figure 3: Effect of aldosterone

which generates a lumen-negative stimulus for potassium efflux through ROMK channels ${ }^{2,6}$ (Figure 3).

The DCT reabsorbs about $10 \%$ of filtered magnesium $\left(\mathrm{Mg}^{2+}\right)$ and is the primary site of active transcellular $\mathrm{Mg}^{2+}$ reabsorption. Apical $\mathrm{Mg}^{2+}$ transport is mediated by transient receptor potential cation channel subfamily M member 6 (TRPM6), a voltagedriven divalent cation channel that is expressed in the early and late DCTs. TRPM6 is also a member of the transient receptor potential channel superfamily, just like TRPV5 and 6. TRPM6 has a 5 -fold preference for magnesium ions over calcium ions, and these transport characteristics allow it to effectively function as a magnesium channel. ${ }^{2}$

Relatively little is known about other transport processes that facilitate transcellular $\mathrm{Mg}^{2+}$ reabsorption. With regards to basolateral $\mathrm{Mg}^{2+}$ transport, a mechanism exists for the reclamation of magnesium back into the peritubular fluid and bloodstream but, the precise molecular identities of these transport processes remain obscure. ${ }^{2}$

\section{Collecting ducts}

The distal tubules join to form CDs, which are about $20 \mathrm{~mm}$ long. There are two main cell types in this region: principal cells and intercalated cells. ${ }^{1}$

\section{Intercalated cells}

Intercalated cells are interspersed among the more numerous principal cells and are essential in the response to acid base status. They help dispose of acid that is generated by dietary intake and cannot be eliminated via the lungs. Here the hydrogen ion secretion is relatively independent of sodium in the tubular lumen (unlike in the PCT). ${ }^{1,7}$

The PCT is extremely important for acid base homeostasis; by recovering filtered bicarbonate $( \pm 90 \%$ of bicarbonate is reabsorbed there). The $\mathrm{CD}$ relies mostly on their intercalated cells to reabsorb the normally smaller amount of residual bicarbonate. In addition, intercalated cells participate in the excretion of ammonia. ${ }^{1,7}$
Intercalated cells are also firmly established as important contributors to collecting duct sodium chloride trans-epithelial transport and are therefore important in the protection of intravascular volume in concert with principal cells.?

Three types of intercalated cells are traditionally recognised, based largely on cell morphology and localisation, namely type $A$, type $B$, and non-A non-B intercalated cells. ${ }^{1,7}$ All intercalated cells express carbonic anhydrase, a metalloenzyme that reversibly catalyses the hydration of $\mathrm{CO}_{2}$ leading to the formation of bicarbonate in their cytoplasm.?

\section{Type A intercalated cells}

Type A intercalated cells can secrete $\mathrm{H}^{+}$into urine. Carbon dioxide $\left(\mathrm{CO}_{2}\right)$ from the blood enters the cell and combines with water $\left(\mathrm{H}_{2} \mathrm{O}\right)$, a reaction catalysed by carbonic anhydrase II to form bicarbonate $\left(\mathrm{HCO}_{3}{ }^{-}\right)$and hydrogen $\left(\mathrm{H}^{+}\right){ }^{1,7}$

During acidosis $\mathrm{H}^{+}$is then excreted into the tubular lumen via the $\mathrm{H}^{+}$-ATPase or the $\mathrm{H}^{+} / \mathrm{K}^{+}$-ATPase pumps at their apical membrane. The latter pump exchanges one potassium ion for each extruded proton/hydrogen. The function of the $\mathrm{H}^{+}$-ATPase pump and $\mathrm{H}^{+} /$ $\mathrm{K}^{+}$-ATPase pump is affected by the $\mathrm{pH}$ difference across the apical membrane and the transepithelial potential difference. During acidosis activity of both $\mathrm{H}^{+}$-ATPase and $\mathrm{H}^{+} / \mathrm{K}^{+}$-ATPase pumps is increased. ${ }^{7}$ Aldosterone augments the $\mathrm{H}^{+}$-ATPase.

Ammonia is also secreted into the lumen by intercalated $A$ cells via specialised transporters. The $\mathrm{H}^{+}$ions in the lumen react with either phosphate $\left(\mathrm{HPO}_{4}{ }^{2-}\right)$ or ammonia $\left(\mathrm{NH}_{3}\right)$. This prevents the $\mathrm{H}^{+}$ions from re-entering the cell, as both new compounds $\left(\mathrm{NH}_{4}{ }^{+}\right.$and $\left.\mathrm{H}_{2} \mathrm{PO}_{4}^{-}\right)$are charged. Hence, they are not able to travel back across the membrane, and so are excreted. ${ }^{5,7}$

$\mathrm{HCO}_{3}{ }^{-}$crosses the basolateral membrane into the interstitium via a bicarbonate chloride $\left(\mathrm{HCO}_{3}{ }^{-} / \mathrm{Cl}^{-}\right)$exchanger channel. To prevent an accumulation of chloride ions and potassium ions within the cell, a $\mathrm{K}^{+} / \mathrm{Cl}^{-}$symporter on the basolateral membrane allows leakage of these ions back into the extracellular fluid ${ }^{7}$ (Figure 4).

The Type A cells thus counteract a metabolic acidosis.

\section{Type B intercalated cells}

The type $\mathrm{B}$ intercalated cells express a $\mathrm{HCO}_{3}{ }^{-} / \mathrm{Cl}$ exchanger (pendrin), at their apical membrane and express $\mathrm{H}^{+}$-ATPase at their basolateral membrane. Once again $\mathrm{CO}_{2}$ enters the cell and combines with $\mathrm{H}_{2} \mathrm{O}$ to form $\mathrm{HCO}_{3}{ }^{-}$and $\mathrm{H}^{+} .{ }^{1,7}$

During alkalosis the $\mathrm{HCO}_{3}-\mathrm{Cl}^{-}$exchanger excretes $\mathrm{HCO}_{3}{ }^{-}$into the tubular lumen in exchange for $\mathrm{Cl}^{-} \mathrm{Cl}^{-}$then leaves the cell via a basolateral transporter to enter the interstitium. $\mathrm{H}^{+}$is then secreted into the interstitium via the $\mathrm{H}^{+}$-ATPase or the $\mathrm{H}^{+} / \mathrm{K}^{+}$ATPase pumps at their basolateral membrane ${ }^{7}$ (Figure 4).

The Type B cells thus counteract a metabolic alkalosis. 


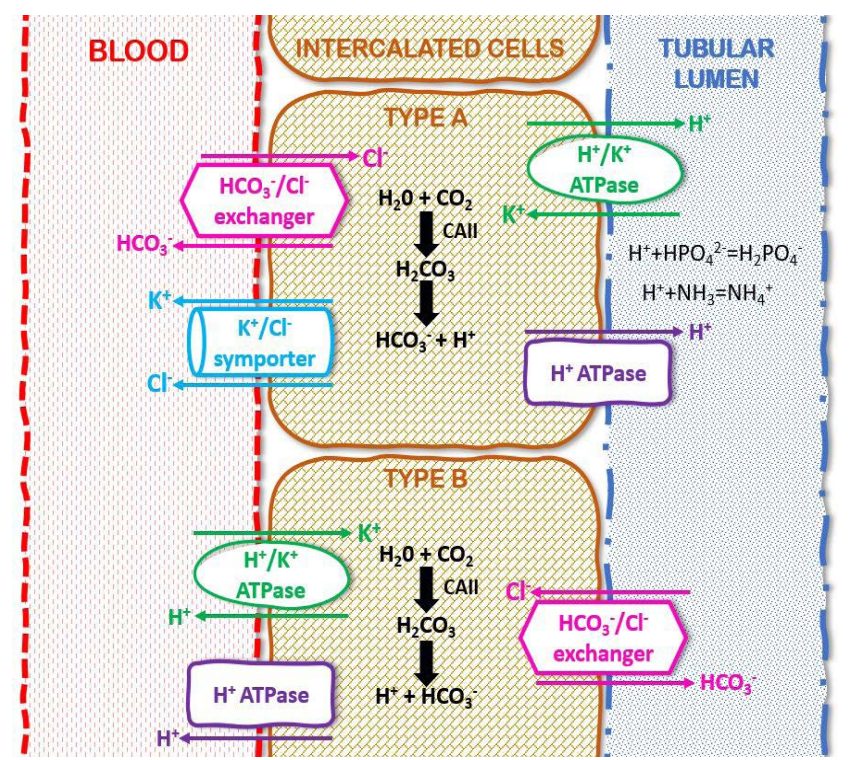

Figure 4: CD cells

Type B cells also play an important role in blood pressure regulation through pendrin mediated chloride uptake, which leads to vascular volume expansion.?

\section{Principal cells}

Principal cells are essential in the control of plasma $\mathrm{Na}^{+}$and $\mathrm{K}^{+}$concentrations, extracellular fluid volume, and BP in most vertebrates, through regulation of $\mathrm{ENaC}$ by aldosterone (see above), and aquaporin 2 by antidiuretic hormone (ADH) or arginine vasopressin. ${ }^{4,6}$

$\mathrm{ADH}$ gets produced by the hypothalamus and is stored in the posterior pituitary gland and released in response to a high plasma osmolality and angiotensin II. ${ }^{1,4,6} \mathrm{ADH}$ then binds to a receptor on the principal cell and activates protein kinase A via a second messenger system, which then causes the insertion of aquaporin II channels into the apical membrane from intracellular vesicles. $\mathrm{H}_{2} \mathrm{O}$ can now enter the principal cells from the tubular lumen via the aquaporin II channels from an

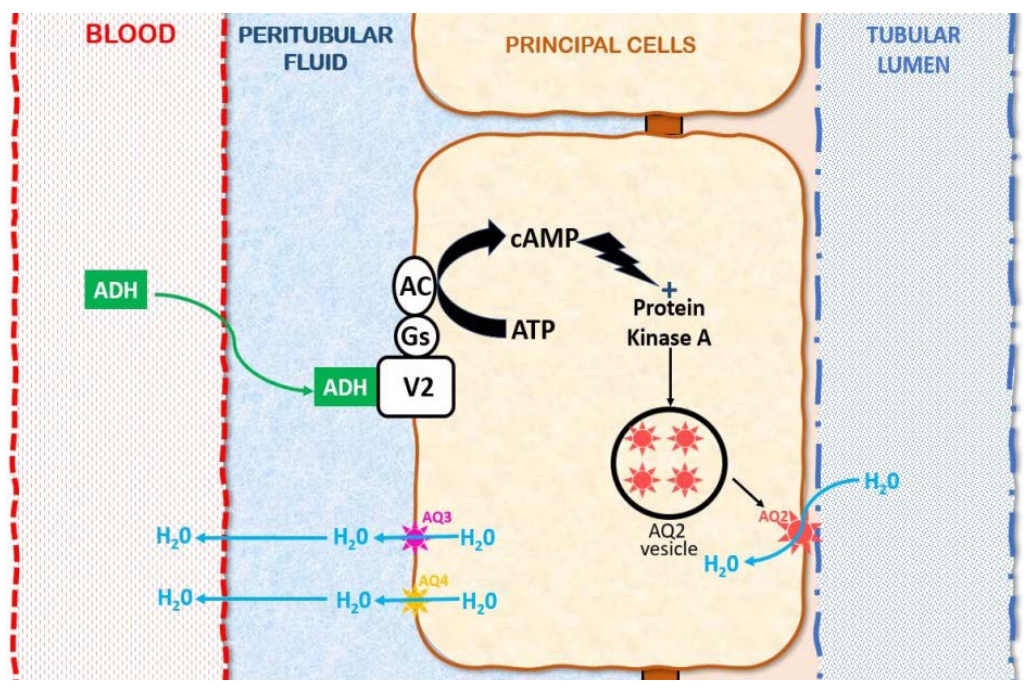

Figure 5: Action of ADH area of low concentration to an area of high concentration. The collecting tubule cells have tight junctions and thus are virtually impermeable to water in the resting state unless $A D H$ is present making them water-permeable and allowing the re-absorption of large amounts of water. ${ }^{4}$

This movement of $\mathrm{H}_{2} \mathrm{O}$ is dependent on the hypertonicity of the medullary fluids (established by a functioning counter-current multiplier and exchanger) that establishes the gradient that draws water out of the collecting tubules. Water reabsorption that depends on the presence of $A D H$ is called 'facultative water reabsorption'. The $\mathrm{H}_{2} \mathrm{O}$ in the principal cells now exit the cells via aquaporin III and IV channels and enter the interstitium ${ }^{4}$ (Figure 5).

$\mathrm{ADH}$ is thus able to control the loss or retention of water without any electrolytes (free water), unlike in other parts of the nephron where water absorption is linked to solute absorption. By controlling free water movement $\mathrm{ADH}$ is able to control ECF osmolality. ${ }^{4}$

$\mathrm{ADH}$ also makes the cells in the medullary CD permeable to urea, which then helps to make the medulla even more hypertonic. Urea then gets reabsorbed into the tubule. This is called urea recycling. ${ }^{1,5}$

Maintaining cardiorenal homeostasis by regulating fluid volume is an important aspect of the cardioprotective properties of atrial natriuretic peptide (ANP), produced primarily by myocytes of the heart atria and released in response to atrial stretch and high systemic blood pressure. In principal cells, ANP inhibits ENaC, thereby contributing to its natriuretic properties. It also inhibits renin secretion and aldosterone production from the adrenal gland thus regulating blood pressure. ${ }^{4}$

Cells in the collecting ducts also secrete drugs, toxins and creatinine. ${ }^{1,4}$

Urine has now been created, and it flows out of the collecting tubules into the renal pelvis and then into the ureter.

\section{Conflict of interest}

The author declares no conflict of interest.

\section{Funding source}

None.

\section{Acknowledgement}

The author would like to thank Dr KD Jivan for creating the diagrams.

\section{ORCID}

\section{KK Purbhoo iD}

https://orcid.org/0000-0002-9001-6764 


\section{References}

1. Kim EB, Susan MB, Scott B, Heddwen LB. Renal function \& micturition. Ganong's review of medical physiology. 25th ed. Lange: McGraw-Hill; 2016. p. 670-5.

2. Subramanya AR, Ellison DH. Distal convoluted tubule. Clin J Am Soc Nephrol. 2014 Dec 5;9(12):2147-63. https://doi.org/10.2215/CJN.05920613.

3. Biga IN, Dawson S, Harwell A, et al. 25.3 Physiology of urine formation: overview. In: Anatomy \& physiology [Internet]. OpenStax/Oregon State University; 2019 [cited 2020 Sep 26]. Available from: https://open.oregonstate.education/aandp/ chapter/25-3-physiology-of-urine-formation-overview/.

4. Pearce D, Soundararajan R, Trimpert $C$, et al. Collecting duct principal cell transport processes and their regulation. Clin J Am Soc Nephrol. 2015 Jan 7;10(1):135-46. https://doi.org/10.2215/CJN.05760513.
5. Ion absorption in the distal convoluted tubule and collecting duct [Internet]. TeachMePhysiology. [cited 2020 Sep 26]. Available from: https:// teachmephysiology.com/urinary-system/nephron/ion-absorption-distalconvoluted-tubule-collecting-duct/.

6. Scott JH, Menouar MA, Dunn RJ. Physiology, Aldosterone. In: StatPearls [Internet]. Treasure Island (FL): StatPearls Publishing; 2020 [cited 2020 Sep 27]. Available from: http://www.ncbi.nlm.nih.gov/books/NBK470339/.

7. Roy A, Al-bataineh MM, Pastor-Soler NM. Collecting duct intercalated cell function and regulation. Clin J Am Soc Nephrol. 2015 Feb 6;10(2):305-24. https:// doi.org/10.2215/CJN.08880914. 